\title{
An Unified Approach for fMRI-Measurements used by a New Real-Time fMRI Analysis System
}

\author{
Maurice Hollmann ${ }^{1}$, Tobias Moench ${ }^{1}$, Claus Tempelmann ${ }^{2}$, \\ Johannes Bernarding ${ }^{1}$ \\ ${ }^{1}$ Institute for Biometry and Medical Informatics, University Magdeburg \\ ${ }^{2}$ Clinic for Neurology II, University Magdeburg \\ Email: maurice.hollmann@medizin.uni-magdeburg.de
}

\begin{abstract}
Real-time functional MRI (rfMRI) offers new experimental paradigms, such as biofeedback and interactive experiments. Usually, several separated software systems are used to control the MRI measuring sequence, the stimulus presentation, and the statistical analysis, which leads to problems concerning the user communication and synchronisation and interaction of the different software systems. Here, an approach is developed which helps to overcome those difficulties by utilising a uniform parameter management using a flexible parameter description that can be used simultaneously by different separated software systems. This approach is used to control two modules: a realtime fMRI application which extracts the current activation, and an analysis-system which evaluates the current brain activation to influence the stimulus presentation and provide feedback to the volunteer.
\end{abstract}

\section{Introduction}

The real-time analysis of brain activation using functional MRI data offers a wide range of new experiments such as investigating self-regulation, bio-feedback or learning strategies [1]. However, besides special data acquisition and realtime data analysing techniques such examination requires dynamic and adaptive stimulus paradigms and selfoptimising MRI-sequences. This paper presents an approach that enables the unified handling of parameters influencing the different software systems involved in the acquisition and analysis process. By developing a custom made Experiment Description Language (EDL) this concept is used for a fast and flexible software environment which treats aspects like extraction and analysis of activation as well as the modification of the stimulus presentation. Furthermore we describe how activation is extracted in real-time using the EDLapproach. The results showed that the developed system in combination with EDL is able to reliably detect and evaluate activation patterns in real-time. With a processing time for data analysis of about one second the approach is only limited by the natural time course of the hemodynamic response function of the brain activation. 
Fig. 1. EDL is the central information repository, which controls the fMRI experiment

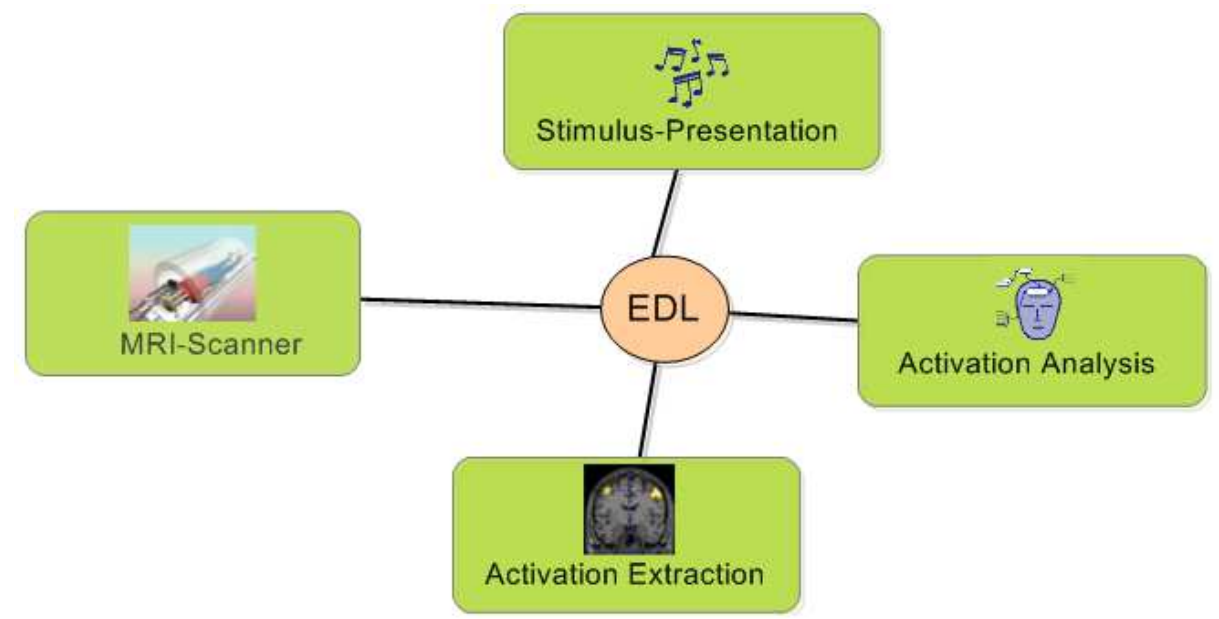

\section{State of the art and new contribution}

Compared to the vast fMRI literature only few experiments were reported for rfMRI $[2,3,4]$. The presented analysis-systems are using no external parameterdescriptions. As usual in fMRI-Experiments the user has to enter all necessary experiment information (e.g. the used stimulus-paradigm) separately for the involved sub-systems, like the MRI acquisition, the stimulus presentation, the activation-extraction and activation analysis. The described disconnected structure leads to an error-proneness concerning input-errors of the user and it complicates the interaction between the different systems. As figure 1 clarifies, the custom made description-language EDL permits a new way to provide essential information to all experimental subsystems. Furthermore the information can be validated and checked for comprehensive consistency. The implemented activation-extraction and -analysis is using the new approach. The whole system is able to perform a fast and unified analysis and classification of real-time fMRI-data.

\section{Methods}

\subsection{System overview}

An EDL-File contains information about the paradigm, which is used to control real-time activation-extraction and -analysis. It may also contain parameters affecting the scan-process. This file is parsed (either in the analysis-system or in the scanner sequence) and the parameters are extracted. More details on the used experiment-description can be found in section "Experiment Description". 
Fig. 2. Structure of an EDL-document: Section statistic

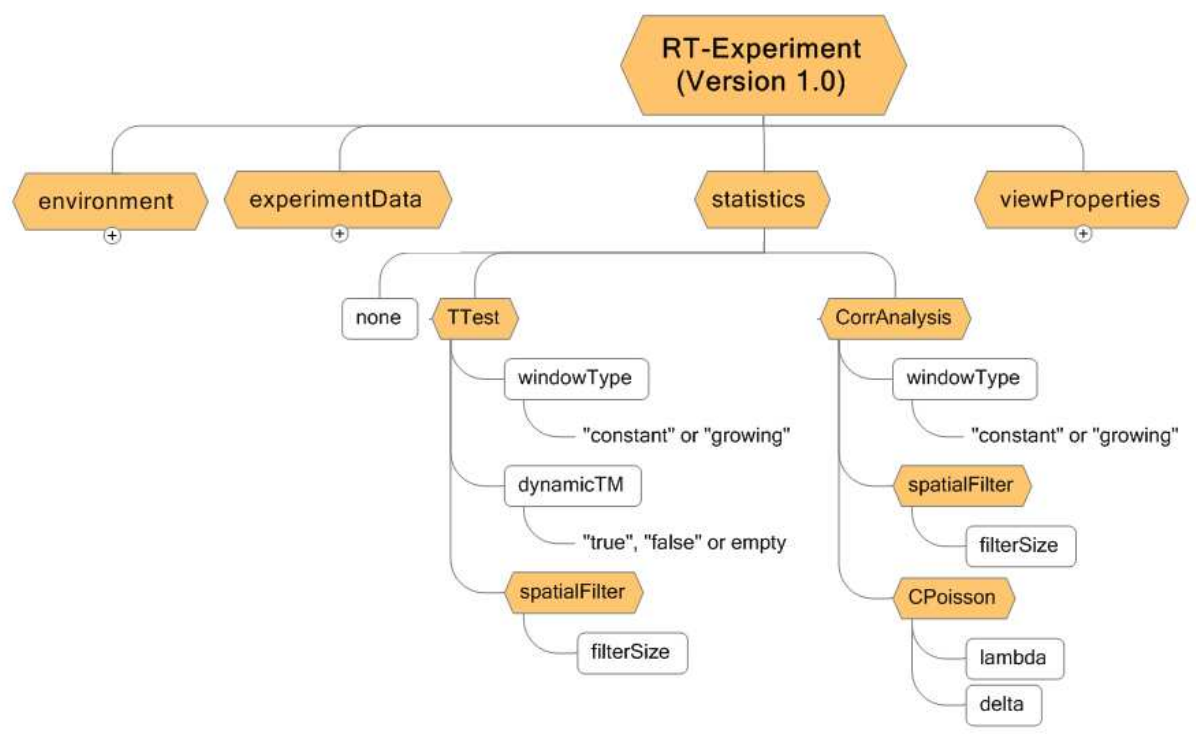

For the realtime-analysis an application was developed, that allows flexible integration of different statistical methods. The developed software modules for activation extraction and activation analysis are implemented in MATLAB, as functions in different packages. The statistical methods of the activation extraction comprehend students t-test and correlation analysis. The visualisation of the statistical results is realised using a maximum-intensity projection on three orthogonal slices (Fig. 3).

\subsection{Experiment description with EDL}

The basis for the description-language EDL is XML. Therefore EDL-forms can be validated using predefined structural information. This structural information is saved as ".xsd" and describes the internal hierarchy as well as parameter limits of the defined elements. As mentioned an EDL-document contains different objects in a hierarchical manner. At the moment an experiment contains four sections:

- environment (environment parameters like data-folders etc.)

- experimentData (paradigm description and functional image modalities)

- statistics (statistical method that should be used and its parameters)

- viewProperties (parameters concerning visualisation)

A representative part of an EDL-document is depicted in figure 2. With the document definition given in a xsd-file, an EDL-document can be validated. For this purpose several tools are available (e.g. OXYGEN, http://www.oxygenxml.com). The validation ensures, that all parameters are within their defined limits, and that the document is syntactically correct. 


\subsection{Scanner sequence and experiments}

The experiments using this approach were performed on a 3 Tesla scanner and a 7 Tesla Scanner (Siemens Medical Systems, Erlangen) at the University of Magdeburg. For the real-time export of the functional data, the sequences on the scanner had to be adapted. The standard epi-sequence and the corresponding reconstruction program (ICE-Program) of Siemens were modified to export every single 3D dataset during the measurement. The sequence parameters were optimized following [5].

Subjects Volunteers were two healthy right-handed males (25 and 28 years). Both gave written consent in participation in our experiments. The study was approved by the ethic committee of the Medical faculty of the University of Magdeburg.

Paradigm and Measurement The volunteers performed right- and left hand finger tapping. The orders for the required action (start, stop, right, left etc.) were given verbally using scanner-compatible headphones and predefined wav-files. After a preparation phase of 40 images ( [5 images baseline, 5 images right tapping, 5 images left tapping] ${ }^{*}$ ) the templates for the activation of the left and the right motor-cortex were derived. These templates were used as input for the activation analysis. The experimental paradigm consisted of a constant block of 20 images: [10 images baseline, 10 images tapping], which was repeated several times in an experimental run. In the experiments the software extracts the activation, analyses the resulting activation maps and automatically classifies which hand was moved by the subject. During the sessions the volunteer was able to explore the visualisation of the activation as well as the result of the activation analysis. The used scan parameters were: $\mathrm{TR}=2000 \mathrm{~ms}, \mathrm{TE}=$ $29 \mathrm{~ms}(3 \mathrm{~T}$ Scanner $) / 20 \mathrm{~ms}(7 \mathrm{~T}$ Scanner $)$, Resolution $=64 \times 64 \times 31(3 \mathrm{~T}$ Scanner $) /$ $64 \times 64 \times 16$ (7T Scanner).

\section{Results}

All experiment parameters were stored in an EDL-document, including the paradigm, preparing-phases and parameters for the activation extraction and -analysis. In that way the experiments were easy and conveniently to plan and to conduct. The activated areas in the presented examples were determined using t-test analysis. Figure 3 shows the statistical result of the 12th image of the constant window (second one after stimulus onset and 4 seconds after beginning of tapping respectively). The subject moved the left hand. Even if the activation could not clearly be identified visually in the MIP-projection, the activation classifier was able to identify correctly which hand had performed the motor action. The software displayed the classification with a string ('Left Hand').

\section{Discussion}

In known studies concerning rfMRI no unified concept connecting the involved systems was used. The new approach of storing parameters for different systems 
Fig. 3. Result of the activation analysis with left hand tapping

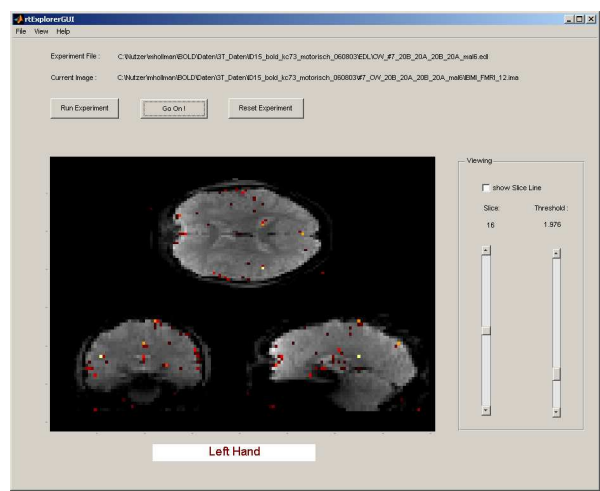

(a) 4s after stimulus-onset

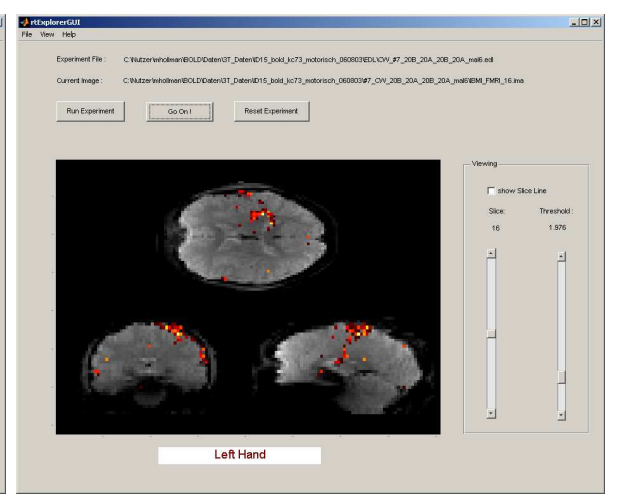

(b) 12s after stimulus-onset

that are involved in real-time fMRI studies in EDL helps to simplify the whole working process. Furthermore it prevents from errors in the parameter design of experiments, because parameter dependencies can be checked automatically. The introduced software system proved to be usable for real-time fMRI studies and integrated control of other applications like activation analysis. The demonstrated influence on stimulus presentation may for example be used to control attention effects in fMRI studies. For further refining the interaction within complex 3D scenes and with the scanner sequence the introduced system can be adapted easily and fast. New methods of activation extraction and the implementation of scan-parameter management trough EDL will serve this process.

\section{References}

1. Weiskopf N, Scharnowski F, Veit R, et al. Physiological self-regulation of regional brain activity using real-time functional magnetic resonance imaging (fMRI). J Physiol 2004;98:357-373.

2. Yoo SS, Fairneny T, Chen NK, et al. Brain-computer interface using fMRI: Spatial navigation by thoughts. Neuroreport 2004;15:1591-1595.

3. Posse S, Binkofski F, Gao K, et al. Real-time fMRI of temporolimbic regions detects amygdala activation during single-trial self-induced sadness. NeuroImage 2003;18:760-768.

4. de Charms RC, Christoff K, Glover GH, et al. Learned regulation of spatially localized brain activation using real-time fMRI. NeuroImage 2004;21:436-443.

5. Posse S, Binkofski F, Schneider F, et al. A new approach to measure single-event related brain activity using real-rime fMRI: feasibility of sensory, motor, and higher cognitive tasks. Human Brain Mapping 2001;12:25-41. 